Military Technical College

Kobry El-Kobbah,

Cairo, Egypt

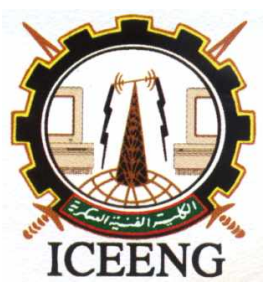

\author{
$6^{\text {th }}$ International Conference \\ on Electrical Engineering \\ ICEENG 2008
}

\title{
Performance Evaluation of Static and Dynamic Location Update and Paging Algorithms for 3G Cellular Networks
}

By

Majdi Salem *

Mahamod Ismail *

Norbahiah Misran *

\section{Abstract:}

Location management plays an important role in 3G-PCS cellular networks. The challenge of supporting rapidly growing numbers of mobile subscribers, while constrained by limited radio spectrum, is being faced by cellular network operators worldwide. In this work a dynamic distance-based location update with sequential paging with no delay constraint algorithm has been considered and compared with other location algorithms in terms of saving radio resources. The location management strategy was implemented in 3G PCS architecture based in cellular systems. The mobility model outputs the cellular parameters and location update messages count during the movement of the mobile station. The dynamic distance-based location update and paging algorithm outperforms the static and other dynamic location update algorithms in terms of the number of location update and paging messages and preserves the channel resources.

\section{Keywords:}

Location management, 3G cellular networks, Static location Update, Dynamic Location Update and Sequential paging.

* Department of Electrical, Electronic and System Engineering, Faculty of Engineering, Universiti Kebangsaan Malaysia, 43600 UKM Bangi, Selangor Darul Ehsan, Malaysia 


\section{Introduction:}

Location management plays an important role in 3G PCS cellular networks. The location management has two major issues, location update and paging. In the location update, the mobile has to inform the network about its position in the network from time to time. The location update process must be as less as possible to save the resources of the network and the battery life of the mobile station. The determination of the locations of the mobile stations in the system has to be easy and simple to implement. In paging, the network has to search for the mobile station by sending signals to the last updated location saved in the network database and detects if the mobile is available to receive the service.

In the existing cellular mobile networks, a two-tier database structure is used to manage the locations of mobile clients. In the system, one of the base stations is defined with a location database, called Home Location Register (HLR) which maintains the client profiles, including the real-time locations of the mobile clients. In addition, each base station (or a group of base stations) also maintains a Visitor Location Register (VLR) to record the mobile clients, which are currently within the cell responsible by the base station [1].

In order to reduce the international/remote roaming signaling traffic, the Gateway Location Register (GLR) within 3G Network is proposed. The GLR is a node between the VLR and the HLR. It handles location management of roaming subscribers in visited network without involving the HLR in every change of LAs. Therefore, the signaling traffic between the visited mobile system and the home mobile system will be reduced and the location updating and the handling of user profile data across network boundaries are optimized. The GLR is located in the visited network. It stores the roamer's information, and handles location management within the network. Note that gateway location registers are optional in the architecture of $3 \mathrm{G}$ cellular networks [2].

\section{Location Management Scheme:}

\section{A. Location and Paging Algorithm}

The mobility model consists of a dynamic location update scheme [3] and sequential paging with no delay constraint scheme [4], [5]. Recent studies suggest that distancebased location update strategies combined with sequential paging could outperform other location management schemes in terms of saving radio resources [6], [7]. A problem has been though those distance-based strategies are in general difficult to implement. The scheme is based on using neighbor cell information, which is normally broadcast on common control channels to enable cell selection of mobile stations. 
The model described in [6] is used for calculating the performance of the mobility model to compare it with some other models in terms of radio resources. Similar random walk models are also reported in [7] - [9].

The performance measures that we consider are the expected number of update messages per slot transmitted by a user, denoted by $\mathrm{U}_{\mathrm{D}}$ and the expected number of searches necessary to locate a user, denoted by $S_{D}$. Obviously, for update and search strategies to be good, both measures should be kept small.

\section{B. Network Topology}

The current personal communication systems (PCS) networks use a static global scheme where the network is divided into zones called Routing Areas (RAs) such that the location uncertainty of a mobile node (MN) is confined to its current RA [10].

The network under the study is consists of one service area with hexagonal cell configuration. Each cell has the same size. Seven location areas, each location area has seven cells construct the target network; the total number of cells in the system is fortyseven as shown in Figure 1. The number of mobile terminals is user defined.

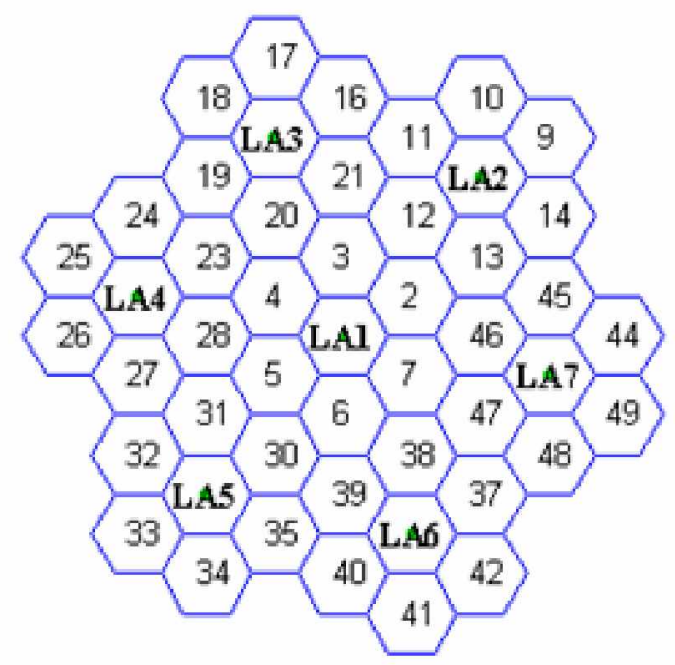

Figure (1): The Used Network Topology

\section{Network Data Architecture}

Simplified network architecture for a $3 \mathrm{G}$ system with the GLR deployment at the edge of the visited networks is shown in Figure 2. 


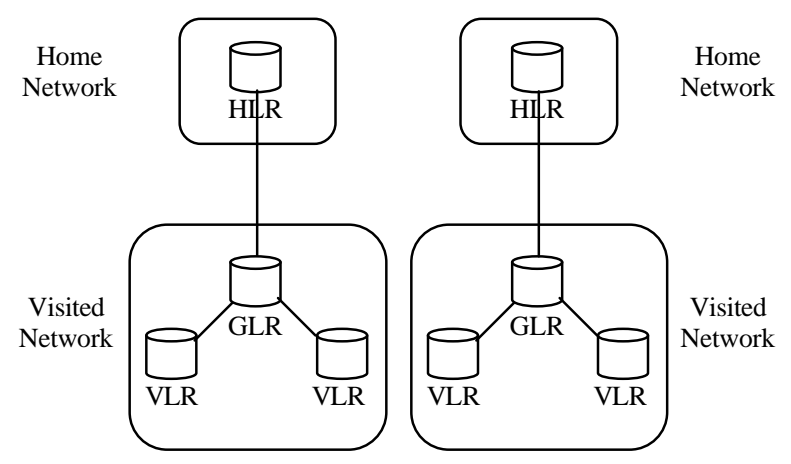

Figure (2): Mobility database architecture

The target network consists of one home location register (HLR) connected to the public switched telephone network (PSTN). This home location register is connected to one gateway location register (GLR), which is connected to four local visitor location registers (VLR). The first visitor location register assigned for the central location area (LA1) because the traffic in this area is maximum. The other visitor location registers had two location areas for each as shown in Figure 3.

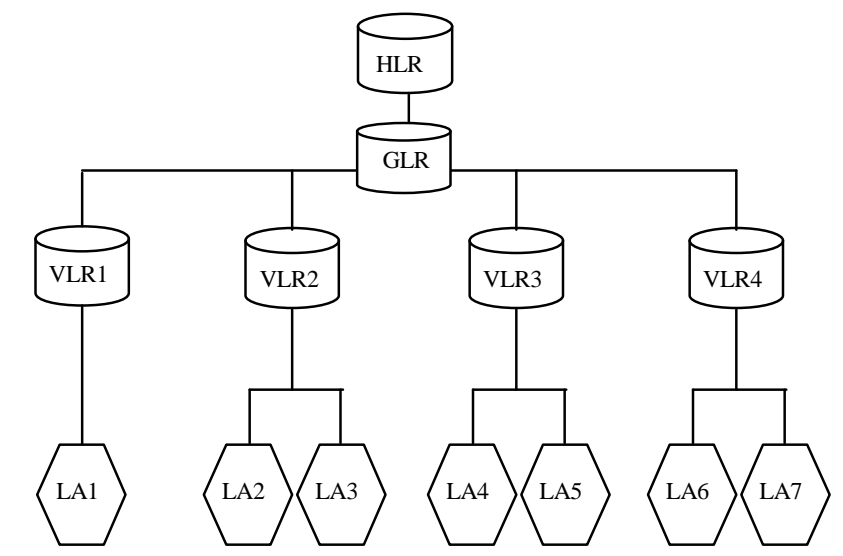

Figure (3): Data Architecture of the Network

\section{Simulation:}

The simulation procedure for location update and paging had been achieved separately as follow:

\section{A. Location Update Algorithm}

The implemented algorithm [3] uses the contact cell and the contact set concept. The contact cell is the cell, in which the mobile station has been last recorded. The contact set contains either only the contact cell or the contact cell together with its neighbor cells. Figure 4 below shows cell 22 as a contact cell. Thus, if the neighbors of 22 are $23, \ldots, 28$ and 22 is the last known cell, then the contact set of the mobile station can be 
either $\{22\}$ or $\{22, \ldots, 28\}$.

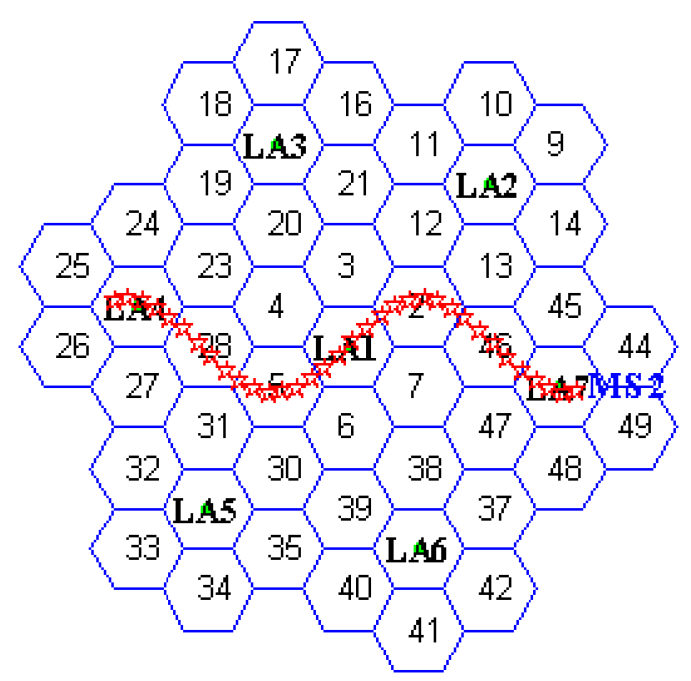

Figure (4): Mobile station routes through hexagonal cells

The principle of the distance-based scheme is the following. Every time the mobile station is in contact with the network, the network stores the contact cell in its location register and the mobile station stores the corresponding contact set. When the mobile station moves within the service area of the network, it performs cell reselection continuously according to the system specifications. Whenever the mobile station selects a new cell, it compares the neighbor cell information of the new cell with its contact set. If the two lists do not have any common cells, the mobile station performs a location update. When the contact set is just the contact cell, this scheme implements the distance-based location update strategy with threshold $d=1$. The previous example used and the scheme gives the following results in Table 1.

Table (1): The Contact Set as One Cell

\begin{tabular}{|c|c|c|c|c|c|c|c|c|}
\hline \multirow[b]{2}{*}{ Initial } & \multirow{2}{*}{\begin{tabular}{|c|} 
Contact Set \\
22
\end{tabular}} & \multicolumn{6}{|c|}{ Neighbors } & \multirow[t]{2}{*}{ Status } \\
\hline & & 23 & 24 & 25 & 26 & 27 & 28 & \\
\hline & 28 & 5 & 4 & 23 & 22 & 27 & 31 & \\
\hline & 5 & 1 & 4 & 28 & 31 & 30 & 6 & Update \\
\hline & 1 & 2 & 3 & 4 & 5 & 6 & 7 & \\
\hline & 2 & 13 & 12 & 3 & 1 & 7 & 46 & Update \\
\hline & 46 & 45 & 13 & 2 & 7 & 47 & 43 & \\
\hline & 43 & 44 & 45 & 46 & 47 & 48 & 49 & Update \\
\hline
\end{tabular}


Let us then consider the same example when the contact set is $\{22, \ldots, 28\}$. Now the mobile station has to compare this list with the list of neighbor cells upon entering a new cell. When the mobile enters cell 28, it compares the new set $\{28,5,4,23,22,27$ and 31 \}. There are four common cells between the new set and the stored one, so no need to update. When mobile station entering cell 5 , it compares the contact set $\{5,1$, $4,28,31,30$ and 6$\}$ with the stored set $\{22,23,24,25,26,27$ and 28$\}$.

These two lists still have 28 as common element and therefore a location update is not needed. When the mobile station finally enters cell 1 , which does not have any of the cells in $\{1, \ldots, 7\}$ common with the stored set, the mobile station performs a location update. The new contact set is $\{1,2,3,4,5,6$ and 7$\}$.

The same procedure achieved with the rest of the routes as shown in Table 2

Table (2): The Contact Set as the cell and its neighbors

\begin{tabular}{|c|c|c|c|c|c|c|c|c|}
\hline & \multicolumn{9}{|c|}{ Contact Set } & Common cells \\
\hline \hline Initial & 22 & 23 & 24 & 25 & 26 & 27 & 28 & 0 \\
\hline & 28 & 5 & 4 & 23 & 22 & 27 & 31 & 4 \\
\hline & 5 & 1 & 4 & 28 & 31 & 30 & 6 & 1 \\
\hline Set 1 & 1 & 2 & 3 & 4 & 5 & 6 & 7 & 0 \\
\hline & 2 & 13 & 12 & 3 & 1 & 7 & 46 & 4 \\
\hline & 46 & 45 & 13 & 2 & 7 & 47 & 43 & 2 \\
\hline Set 2 & 43 & 44 & 45 & 46 & 47 & 48 & 49 & 0 \\
\hline
\end{tabular}

\section{B. Paging Strategy}

The contact set is the set of $\mathrm{n}$ cells; $\mathrm{C}=\{1,2, \ldots, \mathrm{n}\}$ such that the mobile user is guaranteed to be in one of these cells at the time of a call arrival as in Figure 5. A sequential paging scheme is one where the cells in a contact set are partitioned into indexed groups referred to as paging zones on the basis of the cell-wise user location probabilities. The first paging zone consists of the one most probable cell, and the second paging zone consists of the remaining six cells. When a call arrives, cell 1 paged first, and if the user is not located in this cell, then cells 2, 3, 4, 5, 6 and 7 are paged.

\section{Analyses and Discussion:}

One of the most important goals of location management algorithms is efficient utilization of radio spectrum, which is a fixed resource. This requirement can be satisfied by minimizing the number of location updating and paging messages that must be transmitted across the radio interface. 
In the following analysis, we use the same moving mobile stations model with four location schemes (cell-based, LA-based, contact-cell and contact-set). We assume that the mobile station drives in the same route in all the four location schemes. The other system parameters such as cell radius $\mathrm{R}$ and mobile station velocity are the same for all the algorithms. Under those assumptions, we compare the number of location update messages transmitted by the mobile station and the paging achieved by the network. Table 3 shows some results after running the program for the different algorithms with different mobile stations. It shows that the implemented algorithm achieves the best results in terms of number of location update messages. It can be seen that sometimes the LA-based algorithm gives approximately the same results as the implemented algorithm.

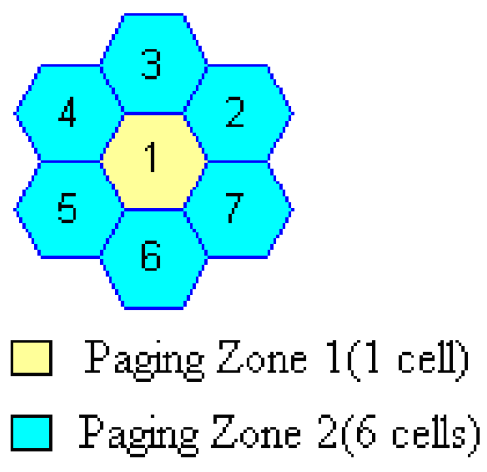

Figure (5): The contact set and paging zones

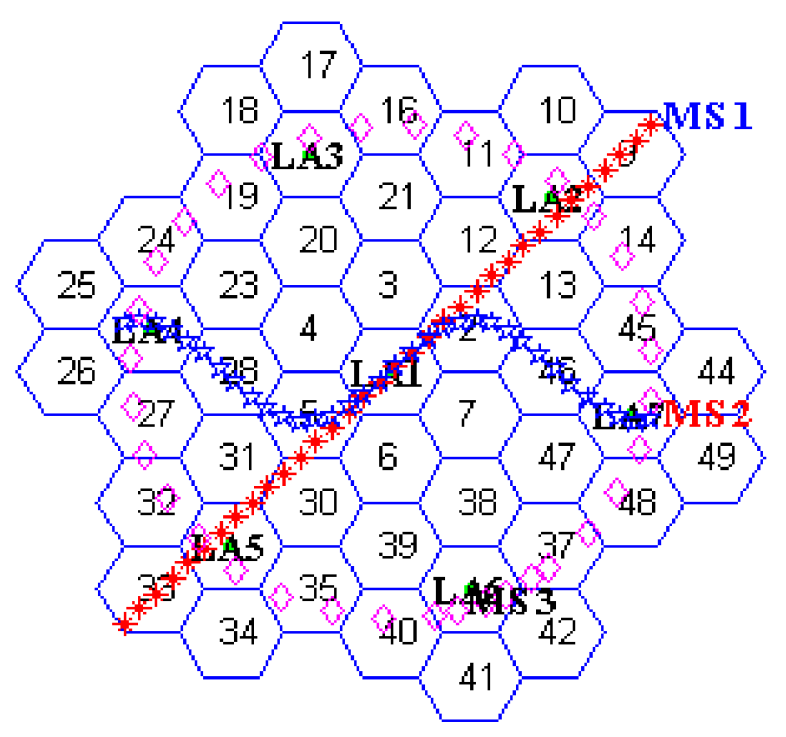

Figure (6): The Route of three mobile stations (MS) traveling through the coverage area 
Table (3): Number of Location Updates for Three Users With Cell Radius ( $R=1 \mathrm{~km})$

\begin{tabular}{|c|c|c|c|c|}
\hline & \multicolumn{4}{|c|}{ Number of Location Updates for: } \\
\hline \hline User & Cell Based & LA Based & Contact Cell & Contact Set \\
\hline $\mathbf{1}$ & 10 & 3 & 4 & 2 \\
\hline $\mathbf{2}$ & 7 & 3 & 3 & 2 \\
\hline $\mathbf{3}$ & 20 & 7 & 9 & 6 \\
\hline
\end{tabular}

\section{Results:}

Theoretically, the expected number of updates $U_{D}$ and polling messages $S_{D}$ performed by the network had been derived in [6]. The calculated results are shown in Table 4 with different values of the movement probability (p).

$\mathrm{U}_{\mathrm{D}}=\mathrm{pQ}_{\mathrm{D}-1}=\frac{2 \mathrm{p}}{\mathrm{D}^{2}}$

$\mathrm{S}_{\mathrm{D}}=1+\sum_{\mathrm{d}=1}^{\mathrm{D}-1} \mathrm{dQ} \mathrm{d}_{\mathrm{d}}=1+\frac{\mathrm{D}}{3}-\frac{1}{3 \mathrm{D}}$

where,

$\mathrm{Q}$ is the stationary probability distribution the distance.

$\mathrm{D}$ is the distance between the new and the last update positions.

In Table 4 the cell-based location update logarithm performs the lowest number of paging messages. But the expected number of update messages is the highest compared to the other algorithms.

Table (4): The expected number of update and searching messages to locate the mobile station

\begin{tabular}{|c|c|c|c|c|}
\hline $\mathbf{P = 1 / 6}$ & Cell Based & LA Based & Contact Cell & Contact Set \\
\hline Threshold Distance & 1 & 3 & 2 & 4 \\
\hline $\mathbf{U}_{\mathbf{D}}$ & 0.34 & 0.04 & 0.09 & 0.02 \\
\hline $\mathbf{S}_{\mathbf{D}}$ & 1 & 1.89 & 1.5 & 2.25 \\
\hline
\end{tabular}

The simulated results for the number of update and paging of the four algorithms are show in Figure 7 . We notice here that the contact set algorithm gives the best relationship between the number of update and search messages. 


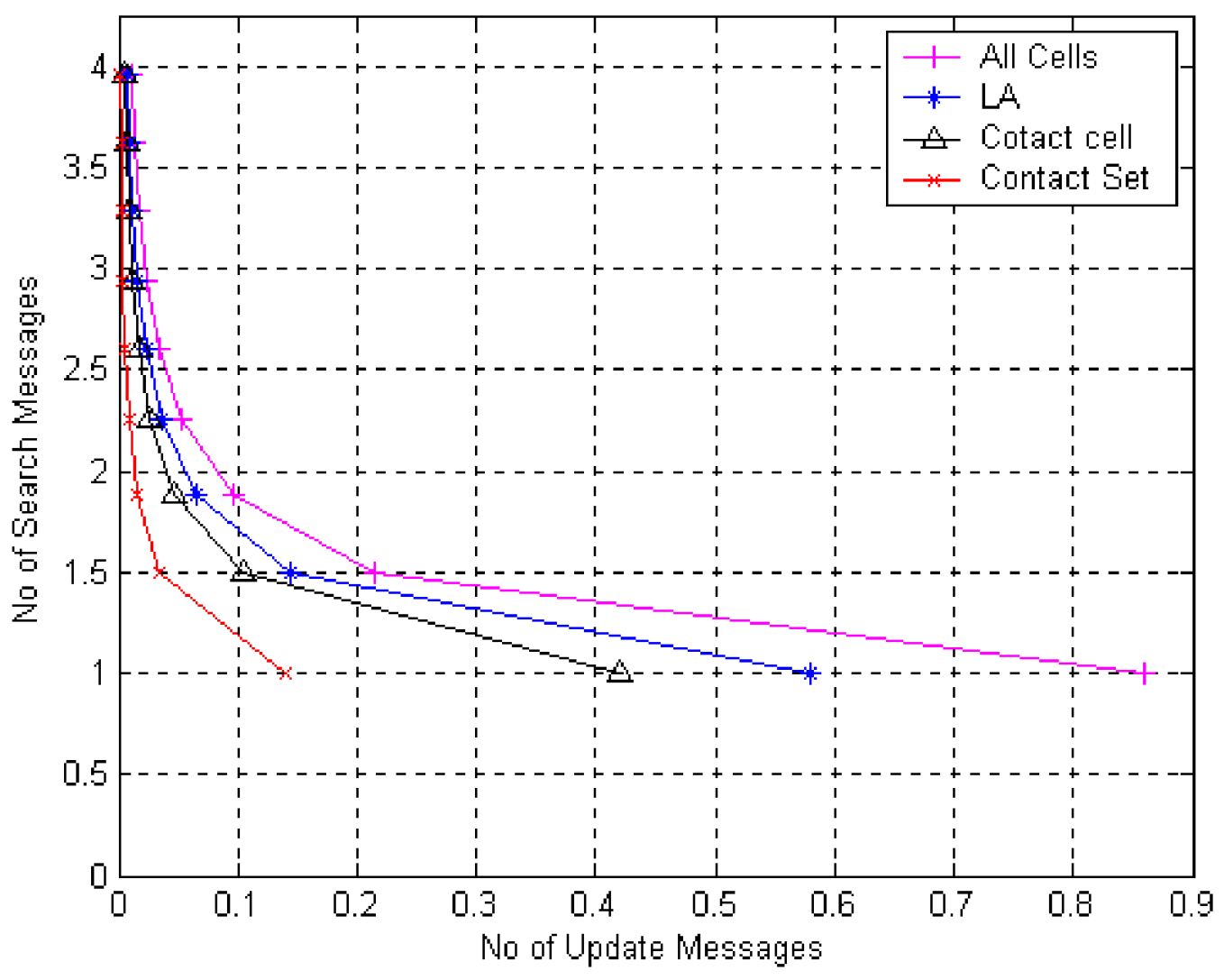

Figure (7): The number of update vs. search messages for one mobile station

\section{Conclusion:}

We presented performance evaluation and comparison of the proposed schemes with the previous schemes in $2 \mathrm{G}$ cellular networks where the GLR is not present

A dynamic distance based location update algorithm was implemented, which dynamically reduced the number of location update messages and outperforms the current location update algorithms in terms of the total number of update messages and save the radio resources of the system. The contact set for the mobile station was created according to the neighbor cell information in the common control channels of the network. The sequential paging algorithm was also implemented to reduce the average paging signals. A trade-off between the number of location update and paging messages has to be taken. 


\section{Acknowledgment:}

This work was supported in part by the project no. 01-01-02-SF0471 through the Ministry of Science, Technology and Innovation Malaysia (MOSTI).

\section{References:}

[1] G. H. Li, K-U Lam and T-W Kuo, Location Update Generation in Cellular Mobile Computing Systems, Proceedings of 15th International Parallel and Distributed Processing Symposium, P. 1017 - 1023, April 2001.

[2] Y. Xiao, Y. Pan and J. Li, Design and Analysis of Location Management for $3 G$ Cellular Networks, IEEE Transactions on Parallel and Distributed Systems, Vol. 15, No. 4, P. 339 - 349, April 2004.

[3] V. Markku, Simple Implementation of Distance-Based Location Updates, IEEE 6th Int. Conf. on Universal Personal Communications. Vol. 1, P. 163-167. October. 1997.

[4] B. Krishnamachari, R-H Gau, S. B. Wicker and Z. J. Haas, Optimal Sequential Paging in Cellular Networks, ACM/Baltzer Wireless Networks, 2003.

[5] Yi-hua Zhu and V.C.M. Leung, Optimization of sequential paging in movementbased location management based on movement statistics, IEEE Transactions on Vehicular Technology, Vol.56, No.2, P. 955-964, March 2007.

[6] B. -N. Amotz, I. Kessler, and M. Sidi, Mobile Users: To Update or not to Update? IEEE INFOCOM Proc., Vol. 2, P. 570-576, June 1994.

[7] U. Madhow, M. L. Honig and K. Steiglitz, Optimization of Wireless Resources for Personal Communications Mobility Tracking, IEEE/ACM Trans. Net., Vol. 3. Vo. 4, P. 698-707, December 1995.

[8] J. S. M. Ho and I. F. Akyildiz, Mobile User Location Update and Paging under Delay Constraints, ACM-Baltzer J. Wireless Networks, Vol. 1. No. 4, P. 413425, December 1995.

[9] K-H Chiang and N. Shenoy, A 2-D Random-Walk Mobility Model for LocationManagement Studies in Wireless Networks, IEEE Transactions on Vehicular Technology, Vol. 53, No. 2, March 2004.

[10] R. Abhishek, M. Archan and K. D. Sajal, Location Update versus Paging TradeOff in Cellular Networks: An Approach Based on Vector Quantization, IEEE Transactions on Mobile Computing, Vol. 6, No. 12, P. 1426-1440, December 2007. 